\title{
Rheumatoid "pneumoconiosis" (Caplan's syndrome) in an asbestos worker: a 17 years' follow-up
}

\author{
I A GREAVES ${ }^{1}$ \\ From the Department of Medicine, Prince Henry Hospital, Sydney, A ustralia
}

Development of rheumatoid pneumoconiosis (Caplan's syndrome) in asbestos workers is rare. One of the earliest reports (Tellesson, 1961) described rheumatoid lung nodules in an asbestos worker with no other initial signs of rheumatoid disease. This report outlines the subsequent clinical course and necropsy of that patient.

\section{Case report}

In 1959 this 39-year-old man suffered a left-sided Staphylococcal pneumonia. He had a 15-year history of work in an asbestos cement factory. Chest radiographs showed circumscribed nodules in the right upper and middle lobes that persisted after recovery from the pneumonia; films in 1953 and 1955 had been normal. He had been treated for diabetes mellitus since 1954

At right thoracotomy in June 1959 nodules were noted in all lobes of the right lung. Several lesions, 0.5 to $4 \mathrm{~cm}$ in diameter, were resected, and some were found to be cavitated. The histological findings were of a central necrotic area surrounded by an intense cellular infiltrate and fibroblasts; most lesions were enclosed in dense fibrosis, and asbestos bodies were observed within the nodules; intervening interstitial fibrosis was noted also. The histology was considered typical of rheumatoid pneumoconiosis related to asbestos exposure. Total asbestos exposure was assessed by Tellesson as not exceeding $5 \times 10^{6}$ particle-years per cubic foot. The patient was not exposed further to asbestos after 1957.

Although absent at the time of thoracotomy, typical rheumatoid arthritis developed within 12 months; the Rose-Waaler titre was $1: 64$ (differential agglutination method; normal, less than $1: 16$ ). Rheumatoid nodules developed over the olecranon bursae and on the dorsum of the right hand. Further nodules developed in the remainder of the right lung and, by September 1961 , in the left lung also; some of these enlarged and cavitated.

In December 1962 an exacerbation of polyarthritis necessitated corticosteroid treatment (oral prednisone, $15 \mathrm{mg}$ daily). Severe bronchopulmonary infection supervened, and several pre-existing cavities showed fluid levels. Tuberculosis was excluded, and this episode of acute infection resolved slowly with postural coughing and prolonged antibiotic treatment. Chronic

'Martin Hardie Research Fellow, Asthma Foundation of New South Wales. sputum production (at least $80 \mathrm{ml}$ daily), purulent or mucopurulent, persisted for the rest of his life, punctuated by acute exacerbations of infection, frequent haemoptyses, and the need for recurrent periods of antibiotics. As the joint symptoms became quiescent, prednisone was ceased after 1963. A fresh crop of pulmonary nodules appeared in the left lung, but no new lesions appeared after 1964. Most nodules increased slowly in size over the years and some developed calcified areas; calcification of the pleura also became more prominent with time (fig 1).

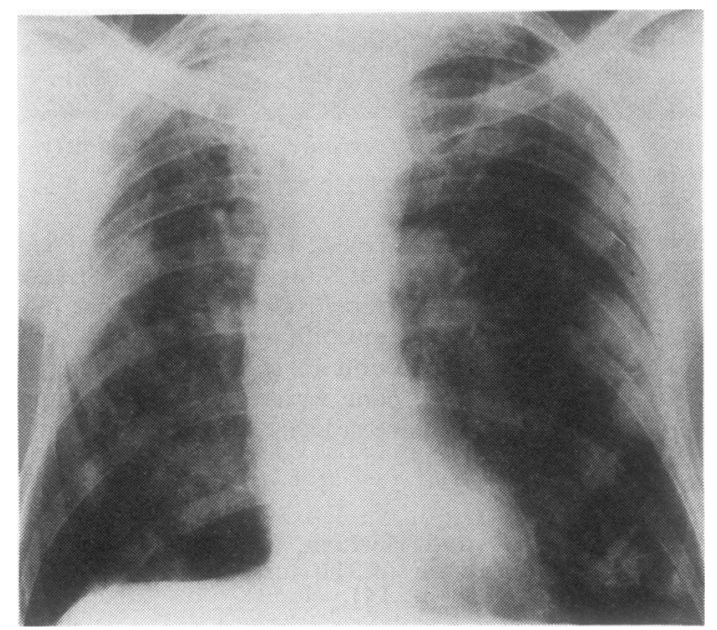

Fig 1 Anteroposterior chest radiograph of 12 July 1974. Numerous large nodular opacities, more prominent on left, are present and several are cavitated. Pleural thickening and calcification are evident; tomography (not shown) showed extensive calcification of the pleura and calcified areas within some of the larger nodules.

In the last seven years of his life he developed splenomegaly, neutropenia, hypochromic normocytic anaemia, and chronic pretibial leg ulcers. Thyrotoxicosis developed in 1972 and was treated with radio- $\mathbb{Q}$ iodine; exophthalmos and ophthalmoplegia developed six months later. Circulating thyroid autoantibodies, $\frac{T}{0}$ antigastric parietal cell antibodies, and antinuclear

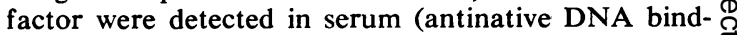
ing $<30 \%$ ); circulating immunoglobulin levels were $\mathbb{D}$ moderately increased (IgG $1900 \mathrm{mg} / \mathrm{dl}$, IgM $320 \mathrm{mg} /$ dl, IgA $670 \mathrm{mg} / \mathrm{dl}$ ). 
Renal impairment developed during 1969. A renal biopsy (1975) showed nephrosclerosis. An extensive anterior myocardial infarction occurred in February, 1976; severe chest pain recurred, and he died suddenly in June.

\section{Necropsy findings}

The pleura was diffusely thickened, fibrosed, and calcified; the lungs were extensively adherent to the chest. Both lungs contained numerous peripheral nodules from 0.5 to $5 \mathrm{~cm}$ in diameter. The right upper lobe contained the largest cavity, which was filled

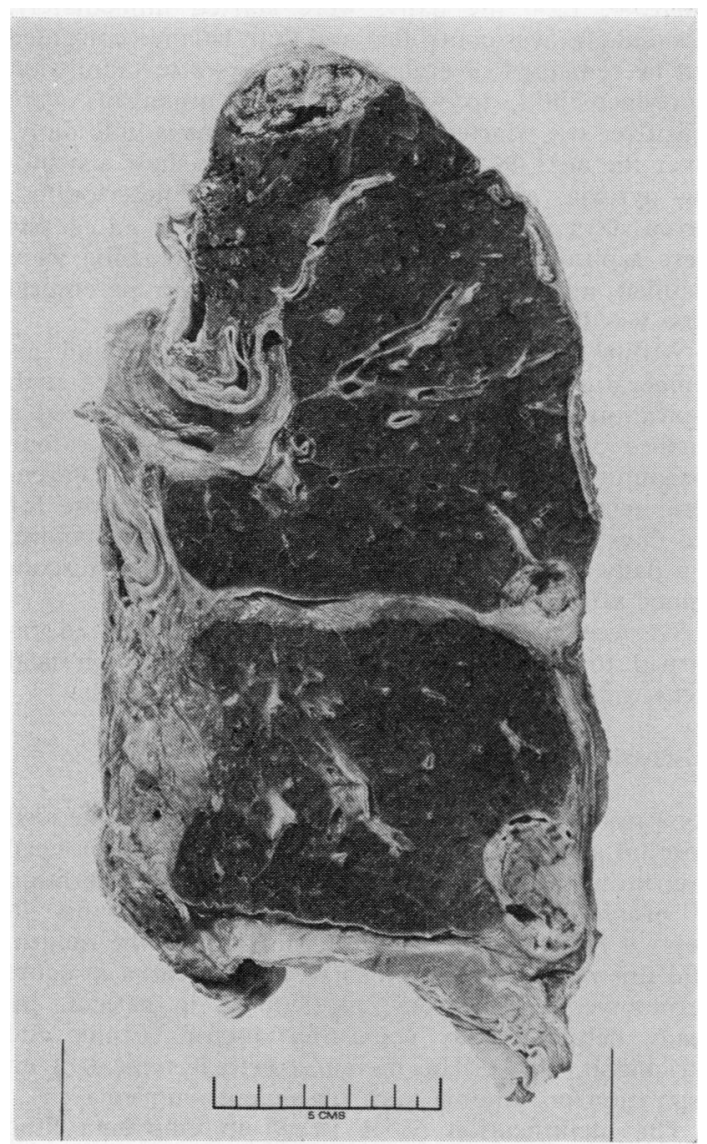

Fig 2 A coronal slice through left lung. Several nodules, 0.5 to $3.0 \mathrm{~cm}$ in diameter, are evident and located subpleurally; cavitation, perinodular fibrosis, and concentric lamellae are variously shown. The pleura is thickened and fibrosed. with darkly pigmented yellow, necrotic material. A similar lesion was present in the right middle lobe. The left lung (fig 2) showed several nodules, 0.5 to 3.5 diameter.

Histology of all nodules was identical to that described and illustrated in Tellesson's report. Occasional ferruginous fragments were seen within nodules, but no typical asbestos bodies. The lung tissue between nodules showed no diffuse fibrotic changes attributable to asbestosis. The heart showed biventricular enlargement, extensive old and recent infarction, and coronary atherosclerosis. Papillary necrosis and focal mesangial glomerulosclerosis were present in both kidneys. The spleen was enlarged $(230 \mathrm{~g})$ but otherwise normal; the liver showed chronic venous congestion. The thyroid was small $(10 \mathrm{~g})$ and fibrotic. There was no evidence of amyloidosis.

\section{Discussion}

In his original description of this patient, Tellesson noted "well-marked interstitial fibrosis"-a feature not found 17 years later when the histological features resembled solely those of multiple chronic rheumatoid nodules. Although Turner-Warwick and Parkes (1970) have reported an association between the extent of radiographic abnormality in asbestos workers and the presence of antinuclear antibodies and rheumatoid factor, exposure to asbestos was possibly of little importance in the development of pulmonary nodules in this patient. Instead, the multiple diseases from which he suffered subsequently, most of which were associated with disturbances of immune function, might suggest an intrinsic susceptibility to immune-mediated lung disease irrespective of asbestos exposure.

I thank Professor B Gandevia and Dr D J Rail for their help, the Department of Medical Illustration, University of New South Wales for the illustrations, and Mrs J Baldwin.

\section{References}

Tellesson, W G (1961). Rheumatoid pneumoconiosis (Caplan's syndrome) in an asbestos worker. Thorax, 16, 372-377.

Turner-Warwick, M, and Parkes, W R (1970). Circulating rheumatoid and antinuclear factors in asbestos workers. British Medical Journal, 3, 492-495.

Requests for reprints to: Dr I A Greaves, Department of Medicine, Prince Henry Hospital, Little Bay, New South Wales, Australia, 2036. 